\title{
Validation of the Name Alteromonas luteoviolacea
}

\author{
M. J. GAUTHIER \\ Centre d'Etudes et de Recherches de Biologie et d'Océanographie Médicale, INSERM Unité 40, 06300, Nice. \\ France
}

\begin{abstract}
The name Alteromonas luteo-violaceus [sic] Gauthier 1976 was not included on the Approved Lists of Bacterial Names and thus has no standing in nomenclature. Therefore, the name is herein validly published, and the original description is amended. The specific epithet has been corrected to "luteoviolacea." The type strain of A. luteoviolacea is CH130 (= ATCC 33492).
\end{abstract}

A group of 16 marine, violet-pigmented bacteria was previously described on the basis of morphological, physiological, and genetic characteristics (4). The cell morphology, Gram reaction, requirement for sodium, inability to grow in the absence of oxygen and to denitrify, and the low guanine-plus-cytosine contents of their deoxyribonucleic acids indicated their inclusion in the genus Alteromonas Baumann et al. (1) as a new species, Alteromonas luteo-violaceus [sic]. However, the name was not validly published because it had not been formally announced in the International Journal of Systematic Bacteriology, and hence it was not included on the Approved Lists of Bacterial Names (9). The purposes of this paper, then, are to effect the valid publication of the name, to correct the specific epithet, and to amend the original description of the organism.

\section{MATERIALS AND METHODS}

Bacterial strains. The 16 strains previously examined (3) (CERBOM Collection numbers $\mathrm{CH} 1, \mathrm{CH} 6$, $\mathrm{CH} 9, \mathrm{CH} 10, \mathrm{CH} 13, \mathrm{CH} 14, \mathrm{CH} 101, \mathrm{CH} 102, \mathrm{CH} 114$, $\mathrm{CH} 115, \mathrm{CH} 116, \mathrm{CH} 122, \mathrm{CH} 123, \mathrm{CH} 124$, and $\mathrm{CH} 130$ ) were isolated from surface seawater in the Mediterranean Sea near Nice, France.

Cultural and biochemical methods. The methods used to determine the cultural and physiological characteristics of the strains were described previously (47).

Utilization of organic compounds. The utilization of 72 organic substrates as sole sources of carbon and energy (see list in Table 1) was determined by the technique of Baumann et al. (2), which included use of their BM Casamino Acids medium.

Electron microscopy. The cells were grown on marine broth (Difco) for $18 \mathrm{~h}$, fixed with glutaraldehyde $(10 \% \mathrm{vol} / \mathrm{vol})$, and then fixed on collodion and shadowed with platinum-palladium (4:1) under vacuum in a Balzers BAF 300 aparatus. Observations and photographs were made with an electron microscope (Hitachi type HU 12) at the Service de Microscopie Electronique de l'Institut Pasteur (Lille, France).

\section{RESULTS}

Cell morphology. All strains consisted of gram-negative, nonsporeforming, nonencapsulated rods which were motile by means of a single polar flagellum (Fig. 1); filamentous forms often occurred in old cultures (over 10 days).

Cultural characteristics. All strains were strict aerobes which formed, after 4 days at $23^{\circ} \mathrm{C}$, circular ( 3 to $5 \mathrm{~mm}$ in diameter), regular, convex, opaque colonies which were more or less violet and which had a pale-violet or yellowish edge of variable width. A few strains $(\mathrm{CH} 10$, $\mathrm{CH} 122$, and $\mathrm{CH} 130$ ) produced colonies with waxy surfaces. Three strains (CH101, CH102, and $\mathrm{CH} 122$ ) developed a cyanide odor (4). Other cultural and physiological characteristics of the strains are summarized in Table 1 . The violet pigment was identified as violacein (4).

Sodium requirements. All strains were unable to grow in the presence of less than $0.1 \mathrm{M} \mathrm{Na}^{+}$; their maximal growth occurred with $0.4 \mathrm{M} \mathrm{Na}^{+}$ (4).

Antibiotic production and catalase effect. Ex cept for strains $\mathrm{CH} 101$ and $\mathrm{CH} 102$, the strains produced a macromolecular polyanionic antibiotic, which was released into the medium whatever the composition of the medium, and one or two autotoxic brominated compounds (8). The strains (except $\mathrm{CH} 101$ and $\mathrm{CH} 102$ ) presented a strong catalase effect (4), as described by Sneath (10). This effect is probably due to the polyanionic antibiotic (3), which is similar to that of $A$. rubra (5), A. citrea (6), and A. aurantia (7).

Metabolism of carbohydrates. Glucose, trehalose, and maltose were oxidatively metabolized (4).

Guanine-plus-cytosine content of deoxyribonucleic acid. The guanine-plus-cytosine contents of the deoxyribonucleic acids ranged from 40.9 to $42.2 \mathrm{~mol} \%\left(T_{m}\right)$, with a mean value for the 16 strains of $41.69 \mathrm{~mol} \%$ (4). 
TABLE 1. Cultural and physiological characteristics of $A$. luteoviolacea, including the type strain, CH130 (= ATCC 33492)

\begin{tabular}{|c|c|c|c|}
\hline \multirow{2}{*}{ Character } & \multicolumn{3}{|c|}{ A. luteoviolacea } \\
\hline & 16 strains & Type strain & Strains which gave negative results \\
\hline \multicolumn{4}{|l|}{ Production of: } \\
\hline Violacein & $14^{a}$ & + & $\mathrm{CH} 13,114$ \\
\hline Yellow pigment & $5(1)^{b}$ & $(+)$ & $\begin{array}{l}\mathrm{CH} 6,9,101,102,114,115,116,122,123,\end{array}$ \\
\hline Brown diffusible pigment & 11 & - & $\mathrm{CH} 101,115,116,122,130$ \\
\hline \multicolumn{4}{|l|}{ Growth at: } \\
\hline $4^{\circ} \mathrm{C}$ & (2) & - & All except $\mathrm{CH} 122,124$ \\
\hline 10 to $30^{\circ} \mathrm{C}$ & 16 & + & \\
\hline $37^{\circ} \mathrm{C}$ & 0 & - & \\
\hline Growth at $\mathrm{pH} 6$ & (7) & - & $\mathrm{CH} 6,10,13,115,116,122,123,124,130$ \\
\hline \multicolumn{4}{|l|}{ Growth at salinity of: } \\
\hline $10 \%$ & (1) & - & All except $\mathrm{CH} 122$ \\
\hline 20 to $40 \%$ & 16 & + & \\
\hline $65 \%$ & (9) & - & $\mathrm{CH} 1,10,13,122,123,124,130$ \\
\hline \multicolumn{4}{|l|}{ Antibiotic produced: } \\
\hline Macromolecular polyanion & 14 & + & CH101, 102 \\
\hline Brominated compounds & 14 & + & CH101, 102 \\
\hline Oxidase & 16 & + & \\
\hline Cytochrome oxidase & 16 & + & \\
\hline Peroxidase & 16 & + & \\
\hline Catalase & 0 & - & \\
\hline Reduction of nitrates & 0 & - & \\
\hline \multicolumn{4}{|l|}{ Hydrolysis of: } \\
\hline Starch & $11(4)$ & + & $\mathrm{CH} 114$ \\
\hline Cellulose & 0 & - & \\
\hline Esculin & 11 & + & $\mathrm{CH} 101,102,114,115,116$ \\
\hline Gelatin & 16 & + & \\
\hline Casein & 16 & + & \\
\hline Indole production & 0 & - & \\
\hline $\mathrm{H}_{2} \mathrm{~S}$ production & 0 & - & \\
\hline Methylene blue reduction & 0 & - & \\
\hline$\beta$-Galactosidase & $\mathbf{0}$ & - & \\
\hline Urease & $\mathbf{0}$ & - & \\
\hline Tryptophan deaminase & 0 & - & \\
\hline Phenylalanine deaminase & 0 & - & \\
\hline Arginine dihydrolase & 0 & - & \\
\hline Glycine decarboxylase & 0 & - & \\
\hline Asparagine decarboxylase & 0 & - & \\
\hline Alanine decarboxylase & $\mathbf{0}$ & - & \\
\hline Lysine decarboxylase & 0 & - & \\
\hline Tween 80 esterase & 16 & + & \\
\hline Tributyrate lipase & 16 & + & \\
\hline Lecithinase & 16 & + & \\
\hline Alkaline phosphatase & 16 & + & \\
\hline Deoxyribonuclease & 16 & + & \\
\hline \multicolumn{4}{|l|}{ Acid from: } \\
\hline D-Glucose & (9) & - & $\mathrm{CH} 101,102,115,122,123,124,130$ \\
\hline D-Mannose & 0 & - & \\
\hline D-Xylose & 0 & - & \\
\hline Trehalose & 16 & + & \\
\hline Fructose & 0 & - & \\
\hline Maltose & 16 & + & \\
\hline Lactose & 0 & - & \\
\hline Saccharose & 0 & - & \\
\hline Mannitol & $\mathbf{0}$ & - & \\
\hline Glycerol & 0 & - & \\
\hline \multicolumn{4}{|l|}{ Susceptibility to: } \\
\hline Vibriostatic agent $0 / 129$ & 0 & - & \\
\hline Polymyxin B & 16 & + & \\
\hline Chloramphenicol & 16 & + & \\
\hline Oleandomycin & 16 & + & \\
\hline
\end{tabular}


TABLE 1 -Continued

\begin{tabular}{|c|c|c|c|}
\hline \multirow{2}{*}{ Character } & \multicolumn{3}{|c|}{ A. luteoviolacea } \\
\hline & 16 strains & Type strain & Strains which gave negative results \\
\hline Erythromycin & 16 & + & \\
\hline Rifamycin & 16 & + & \\
\hline Streptomycin & (2) & - & $\begin{array}{l}\mathrm{CH} 6,9,10,13,14,101,102,114,115,116, \\
\quad 122,123,124,130\end{array}$ \\
\hline Penicillin G & 0 & - & \\
\hline Kanamycin & 0 & - & \\
\hline Tetracycline & 0 & - & \\
\hline \multicolumn{4}{|l|}{ Utilization of: } \\
\hline D-Glucose & (9) & + & $\mathrm{CH} 101,102,115,122,123,124,130$ \\
\hline D-Mannose & 0 & - & \\
\hline D-Xylose & 0 & - & \\
\hline D-Galactose & 0 & - & \\
\hline DL-Arabinose & 0 & - & \\
\hline L-Rhamnose & 0 & - & \\
\hline D-Fructose & 0 & - & \\
\hline D-Ribose & 0 & - & \\
\hline Trehalose & 16 & + & \\
\hline Maltose & 16 & + & \\
\hline Raffinose & 0 & - & \\
\hline Saccharose & 0 & - & \\
\hline Cellobiose & 0 & - & \\
\hline Lactose & 0 & - & \\
\hline Deoxyribose & 0 & - & \\
\hline Melibiose & 0 & - & \\
\hline Starch & $11(5)$ & + & \\
\hline Glycerol & 0 & - & \\
\hline Erythritol & 0 & - & \\
\hline Mannitol & 0 & - & \\
\hline Sorbitol & 0 & - & \\
\hline meso-Inositol & 0 & - & \\
\hline Dulcitol & 0 & - & \\
\hline Adonitol & 0 & - & \\
\hline Esculin & 0 & - & \\
\hline Inulin & 0 & - & \\
\hline Salicin & 0 & - & \\
\hline D-Gluconate & 0 & - & \\
\hline D-Glucosamine & 16 & + & \\
\hline $\mathrm{N}$-Acetylglucosamine & 16 & + & \\
\hline Glucuronate & 0 & - & \\
\hline$o$-Hydroxybenzoate & 0 & - & \\
\hline$m$-Hydroxybenzoate & 0 & - & \\
\hline$p$-Hydroxybenzoate & 0 & - & \\
\hline Deoxycholate & 0 & - & \\
\hline Citrate & 0 & - & \\
\hline$\alpha$-Ketoglutarate & 0 & - & \\
\hline Succinate & 0 & - & \\
\hline Fumarate & 0 & - & \\
\hline DL-Malate & 0 & - & \\
\hline Oxalate & $6(2)$ & + & $\mathrm{CH} 1,6,9,10,11,13,14,114$ \\
\hline Pyruvate & 8 & + & $\mathrm{CH} 1,6,9,13,114,115,116,124$ \\
\hline DL-Lactate & 5 & + & $\mathrm{CH} 1,6,9,10,11,13,114,115,122,123,124$ \\
\hline Acetate & 0 & - & \\
\hline L-Glutamate & 0 & - & \\
\hline Ascorbate & 12 & + & $\mathrm{CH} 6,114,115,116$ \\
\hline Malonate & 0 & - & \\
\hline L-Tartrate & 8 & + & $\mathrm{CH} 1,6,10,11,13,14,115,116$ \\
\hline DL-Glycerate & 0 & - & \\
\hline Tributyrin & 0 & - & \\
\hline Tween 20 & 16 & + & \\
\hline Tween 80 & 16 & + & \\
\hline Cholesterol & 0 & - & \\
\hline Lecithin & 0 & - & \\
\hline
\end{tabular}


TABLE 1 -Continued

\begin{tabular}{|c|c|c|c|}
\hline \multirow{2}{*}{ Character } & \multicolumn{3}{|c|}{ A. luteoviolacea } \\
\hline & 16 strains & Type strain & Strains which gave negative results \\
\hline Creatine & 0 & - & \\
\hline Urea & 0 & - & \\
\hline DL- $\beta$-Hydroxybutyrate & 0 & - & \\
\hline L-Proline & 12 & + & $\mathrm{CH} 1,6,114,116$ \\
\hline L-Asparagine & $14(2)$ & + & \\
\hline L- $\alpha$-Alanine & 16 & + & \\
\hline L-Phenylalanine & 12 & + & $\mathrm{CH} 1,6,114,115$ \\
\hline L-Leucine & 0 & - & \\
\hline Tryptophan & 0 & - & \\
\hline L-Cysteine & (6) & $(+)$ & $\mathrm{CH} 6,9,10,11,13,14,101,122,123,124$ \\
\hline L-Lysine & 6 & + & $\mathrm{CH} 6,9,10,11,13,14,101,122,123,124$ \\
\hline Glycyl-glycine & 16 & + & \\
\hline L-Threonine & $7(9)$ & $(+)$ & \\
\hline L-Histidine & $2(8)$ & $(+)$ & CH6, 9, 10, 101, 122, 123 \\
\hline L-Arginine & (4) & - & $\begin{array}{l}\mathrm{CH} 1,9,10,13,14,101,102,114,115,116, \\
\quad 123,130\end{array}$ \\
\hline L-Ornithine & 0 & - & \\
\hline L-Tyrosine & 6 & + & CH6, 9, 10, 11, 13, 14, 101, 122, 123, 124 \\
\hline
\end{tabular}

"Number of strains which gave a positive result.

${ }^{b}$ Numbers in parentheses are numbers of strains giving a slight reaction.

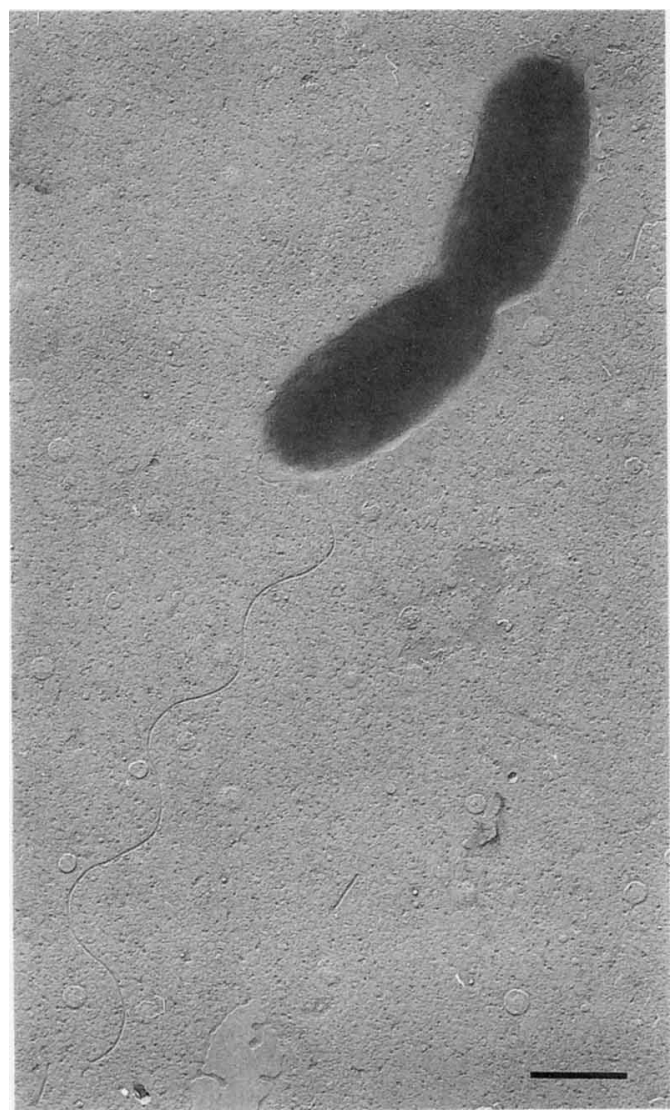

FIG. 1. Electron micrograph of strain $\mathrm{CH} 130$ (= ATCC 33492). Bar equals $1 \mu \mathrm{m}$.

\section{DISCUSSION}

The remarkable similarity of the phenotypic characteristics of the 16 strains investigated led me (4) to consider these strains as belonging to a single species of the genus Alteromonas Baumann et al. The name Alteromonas luteo-violaceus (sic) was proposed for this species (4), but it was not validly published because the name had not been announced in the International Journal of Systematic Bacteriology; furthermore, the type strain of the species had not been designated. Consequently, the name was not included on the Approved Lists of Bacterial Names (9). In addition, the specific epithet was incorrect because the ending did not agree with the gender of the generic name. The specific epithet is therefore here corrected to "luteoviolacea," and the name Alteromonas luteoviolacea is here validated (L. adj. luteus yellow; L. adj. violaceus violet; M.L. adj. luteoviolaceus yellowish violet).

The type strain of A. luteoviolacea is $\mathrm{CH} 130$. This strain has been deposited in the American Type Culture Collection, Rockville, Md., under the number ATCC 33492 and in the National Collection of Marine Bacteria, Aberdeen, Scotland, as NCMB 1893. It was isolated from surface seawater in the neritic zone near Nice, France.

\section{ACKNOWLEDGMENTS}

I thank P. Baumann for his interest and advice in revising this manuscript. I am also grateful to G. Torpier (Service de Microscopie Electronique, Institut Pasteur, Lille, France), who performed the electron microscopy. 


\section{REPRINT REQUESTS}

Address reprint requests to: Dr. M. J. Gauthier, Centre d'Etudes et de Recherches de Biologie et d'Océanographie Médicale, INSERM U 40, 1 avenue Jean Lorrain, 06300. Nice, France.

\section{LITERATURE CITED}

1. Baumann, L., P. Baumann, M. Mandel, and R. D. Allen 1972. Taxonomy of marine eubacteria. J. Bacteriol 110:402-429.

2. Baumann, P., L. Baumann, and M. Mandel. 1971. Taxonomy of marine bacteria: the genus Beneckea. J. Bacteriol. 107:268-294.

3. Gauthier, M. J. 1972. Antagonismes et synergies dans l'antibiose chez certaines bactéries marines. Rôle de certains enzymes respiratoires dans ces phénomènes. Rev. Int. Oceanogr. Med. 26:65-84.

4. Gauthier, M. J. 1976. Morphological, physiological and biochemical characteristics of some violet pigmented bac- teria isolated from seawater. Can. J. Microbiol. 22:138-149.

5. Gauthier, M. J. 1976. Alteromonas rubra sp. nov., a new marine antibiotic-producing bacterium. Int. J. Syst. Bacteriol. 26:459-466.

6. Gauthier, M. J. 1977. Alteromonas citrea, a new gramnegative, yellow-pigmented bacterium isolated from seawater. Int. J. Syst. Bacteriol. 27:349-354.

7. Gauthier, M. J. 1979. A new antibiotic-producing bacterium from seawater: Alteromonas aurantia. sp. nov. Int. J. Syst. Bacteriol. 29:366-372.

8. Gauthier, M. J., and G. N. Flatau, 1976. Antibacterial activity of marine violet pigmented Alteromonas with special reference to the production of brominated compounds. Can. J. Microbiol. 22:349-354.

9. Skerman, V. B. D., V. McGowan, and P. H. A. Sneath (ed.). 1980. Approved lists of bacterial names. Int. J. Syst. Bacteriol. 30:225-420.

10. Sneath, P. H. A. 1956. Cultural and biochemical characteristics of the genus Chromobacterium. J. Gen. Microbiol. 15:70-98. 